\title{
A conspectus of the tribe Andropogoneae of Poaceae in Western Himalaya
}

\author{
Shailja Tripathi, Priyanka Agnihotri ${ }^{1}$, Rekha Yadav, Dileshwar Prasad, \\ Shubham Jaiswal and Tariq Husain \\ Plant diversity, Systematics \& Herbarium Division, CSIR-National Botanical Research Institute, \\ Rana Pratap Marg, Lucknow - 226001, India \\ ${ }^{1}$ Corresponding author;e-mail: priyagni_2006@yahoo.co.in
}

[Received 30.05.2019; Revised 22.06.2019; Accepted 28.06.2019; Published 30.06.2019]

\begin{abstract}
The present work is a critical assessment of published literature, herbarium records and field observations on the diversity, distribution and endemism in the tribe Andropogoneae belonging to the family Poaceae (Grasses) from Western Himalaya. A total of 33 genera with 113 species and one sub-species have been recorded from Western Himalaya. Maximum species diversity has been observed between an elevation of $150062000 \mathrm{~m}$ asl and the most diverse genus is Cymbopogon which is represented by 12 species and one sub-species. The tribe is of enormous economic potential and thereby needs documentation of its diversity and conservation status.
\end{abstract}

Key words: Andropogoneae, Poaceae, diversity, distribution, endemism, Western Himalaya

\section{INTRODUCTION}

Western Himalaya refers to the western half of the Himalayan mountain region, which stretches from northeastern Afghanistan through India to central Nepal. In India, Western Himalaya spreads over three states viz. Jammu and Kashmir, Himachal Pradesh and Uttarakhand, categorized as one phytogeographic unit encompassing an area of about 3.31 lakh square $\mathrm{km}$ which represents ca $10 \%$ of the total geographical area of the country (Jalal \& Jayanthi 2015). Western Himalaya has always been a fascinating spot for the botanists for exploration of biodiversity. Distinctive features of the Western Himalaya render it complexity attributed to both physical as well as biological diversity. The region is bestowed with significant altitudinal and temperature gradient and variable climatic conditions, manifesting biodiversity profusion over there. Presence of valleys, glaciers, continental ridges and dunes adds to the geographical beauty of the region. Western Himalaya possesses vast biodiversity which is still untapped and requires major efforts to explore the region entirely so that new insights can be inferred relevant for nature and mankind. From ancient times, different workers have been involved in the study of the diversity of various life forms present in the Western Himalayan region. In this context, plants have always been the keen centre of attraction alluring the researchers with their beauty. Most of the plants are very showy and beautiful and therefore easily get selected to work upon. Several studies have been made in such type of Himalayan plants including those of Pedicularis (Husain et al. 2010), Aconitum (Agnihotri et al. 2015), Delphinium (Agnihotri \& Husain 2019) and orchids (Jalal \& Jayanthi 2015). Grasses, on the other hand, lack the conspicuous features and therefore, have been overlooked by botanists. Despite the utmost importance of grasses to mankind, it continues to be a neglected subject (Yadav 2010). 
Inconspicuous floral organs, difficult identification, the complicated structure of the spikelets and inflorescence repel the researchers to get in touch with grasses. Taxonomically, grasses are highly complex in nature and require incentives to resolve the complexity of the group. Being one of the regions of biodiversity hot spots, Western Himalaya has an abundance of floristic diversity. Grasses form an integral part of this diversity. Unfortunately, due to the above-specified reasons, grasses are always taken for granted due to which significant studies of grasses have not been undertaken yet. Keeping in mind these aspects and realizing the relevance of grass study, the present study has been designed to frame a picture of biodiversity of a particular tribe Andropogoneae which later would be complemented by the study of the whole group of grasses from the specified region.

The tribe Andropogoneae (Poaceae) nested in the subfamily Panicoideae along with Maydeae and Paniceae is characterized by the presence of fragile racemes bearing spikelets (Clayton 1981). Globally, ca 1000 species belonging to 85 genera of Andropogoneae (Clayton et al. 1986) are known to be widely distributed in the tropics and extend into warm temperates. Hartley in 1950 on the basis of his studies suggested that the Indo-Malayan region has relatively rich species diversity of the group highly concentrated in Western India and Southern Indonesia. In Indian context, the recent studies (Kiran et al. 2008) revealed that Peninsular India especially the region of Western Ghats is considered to be the centre of diversity of Andropogonoid grasses supported by morpho-taxonomic and phytogeographical analysis. Members of Andropogoneae bear paired spikelets with one sessile and other pedicelled, arranged in modified racemes terminating in a triad of one sessile and two pedicelled spikelets (Simon 2007). Most of the Andropogonoids possess a distinctive disarticulating rachis which is responsible for their worldwide distribution (Clayton 1987). The variations in climatic factors considerably influence the distribution of these grasses (Hartley 1950). Extreme morphological variations in the inflorescence and raceme segments of the tribe render it as one of the most complex groups of grasses. The occurrence of inter-generic hybrids has also been reported in the tribe (Pachakkil et al. 2018). The tribe includes several genera of ecological and economic importance distributed worldwide like Saccharum, Sorghum, Cymbopogon, Chrysopogon, etc. Most of the medicinally important and essential oilbearing grasses including Cymbopogon, Dichanthium, Saccharum, Chrysopogon belong to the tribe Andropogoneae (Shukla 1996).

Views regarding the classification of grasses have always been of a controversial nature. Different workers opted distinct classification systems for classifying grasses. The first scientific subdivision of the family was done by Robert Brown in 1814 where he distinguished the spikelets of Pooideae and Panicoideae (Bor 1960). Demarcation in further divisions of the tribe Andropogoneae has been problematic (Vegetti 1998). Hackel in 1889 for the first time provided insights of Andropogoneae where he divided the tribe into five subtribes and 30 genera which are further categorized as series, subgenera and sections. Recent phylogenetic studies revealed the monophyletic origin of Andropogoneae (GPWG 2001). A floristic account of Indian grasses has been detailed by Hooker (1897) in his Flora of British India. Thereafter, an elaborated account of grasses has provided by Bor (1960). Meanwhile, various fragmentary studies on the grasses have been in prevalence. A comprehensive systematic study of grass flora from India is still lacking and this lacuna is to be filled as soon as possible.

The present article is an attempt to provide an overview of the tribe Andropogoneae which can be used as baseline data for further studies of the group. Classification of grasses provided by Bor (1960) has been used as a basis in the present study. 


\section{METHODOLOGY}

For abundance and distribution related information of the taxa, published literature was surveyed followed by the field observation, consultation of some national herbaria viz. CAL, CDRI, CIMAP, DD \& LWG and several plant database websites etc. Based on the information, the abundance was confirmed through ground-truthing conducting sporadic field survey in the Western Himalaya region. Field surveys to the specified region were done during the months of August, September and October 2018 and extensive collections were made from Manali, Rohtang Pass, Solang valley, Naggar region of Himachal Pradesh, Pauri Garhwal, Auli, Joshimath, Srinagar and Nainital of Uttarakhand. Thereafter, identification of the specimens was done using literature from different sources. The keys of identification provided by Bor (1960), Sreekumar (1991), Shukla (1996), Potdar et al. (2012) have been used. Moreover, the herbaria specimens have also been consulted in order to make the identification more authenticated.

\section{RESULTS AND DISCUSSION}

In Western Himalaya, Poaceae is represented by 33 genera with 113 species and one subspecies (Table 1) which presents $52 \%$ of the total genera and $32 \%$ of the total species of Andropogoneae found in India and 39\% of the total genera and 11\% of the species found in the world (Table 2). Our data analysis displays that Uttarakhand has the maximum diversity of the Andropogonoids followed by Himachal Pradesh and Jammu and Kashmir (Figure 1) 44 taxa of Andropogonoids are found to occur exclusively in Uttarakhand as compared to seven in Himachal Pradesh and none in Jammu and Kashmir. As a whole, the region is found to have 30 taxa in common among the three states. The diversity in Uttarakhand may be attributed to its topographical and climatic factors which provide suitable environmental conditions for the luxurious growth of these grasses. But it is worthy to note that diversity of Andropogonoids is found to be more in Peninsular India as compared to the Western Himalaya. This difference in diversity may be attributed to the climatic variations existing between the two regions.

Table 1. Distribution of different taxa of Andropogoneae in Western Himalaya [Abbreviations used: $\mathrm{HP}=$ Himachal Pradesh; J\&K = Jammu \& Kashmir; UK = Uttarakhand]

\begin{tabular}{|l|l|l|l|l|l|}
\hline S. & Name of taxa & $\begin{array}{l}\text { Flowers } \\
\text { No. }\end{array}$ & & $\begin{array}{l}\text { Altitude } \\
(\mathbf{m})\end{array}$ & \multicolumn{2}{|l|}{ Distribution } \\
\cline { 5 - 6 } & & & $\begin{array}{l}\text { Western } \\
\text { Himalaya }\end{array}$ & World \\
\hline 01 & $\begin{array}{l}\text { Andropogon munroi C.B. } \\
\text { Clarke }\end{array}$ & Jul.-Sept. & $1500-3500$ & HP, UK & China, Nepal \\
\hline 02 & $\begin{array}{l}\text { Andropogon pumilus } \\
\text { Roxb. }\end{array}$ & Aug.-Oct. & 5000 & UK & Indo-China \\
\hline 03 & Apluda mutica L. & $\begin{array}{l}\text { May.- } \\
\text { Feb. }\end{array}$ & $1000-2400$ & $\begin{array}{l}\text { HP, J\&K, } \\
\text { UK }\end{array}$ & $\begin{array}{l}\text { Bhutan, China, } \\
\text { Japan, Madagascar, } \\
\text { South East Asia }\end{array}$ \\
\hline 04 & $\begin{array}{l}\text { Apocopis paleacea } \text { (Trin.) } \\
\text { Hochr. }\end{array}$ & Jul.- Nov. & $900-1200$ & UK & $\begin{array}{l}\text { Bhutan, Malaysia, } \\
\text { Thailand }\end{array}$ \\
\hline
\end{tabular}




\begin{tabular}{|c|c|c|c|c|c|}
\hline \multirow{2}{*}{$\begin{array}{l}\text { S. } \\
\text { No. }\end{array}$} & \multirow[t]{2}{*}{ Name of taxa } & \multirow{2}{*}{$\begin{array}{l}\text { Flowers } \\
\text { \& Fruits }\end{array}$} & \multirow{2}{*}{$\begin{array}{l}\text { Altitude } \\
\text { (m) }\end{array}$} & \multicolumn{2}{|c|}{ Distribution } \\
\hline & & & & $\begin{array}{l}\text { Western } \\
\text { Himalaya }\end{array}$ & World \\
\hline 05 & Apocopis vaginata Hack. & $\begin{array}{l}\text { Sept.- } \\
\text { Dec. }\end{array}$ & $300-1200$ & UK & - \\
\hline 06 & $\begin{array}{l}\text { Arthraxon nudus (Steud.) } \\
\text { Hochst. }\end{array}$ & $\begin{array}{l}\text { Aug.- } \\
\text { Feb. }\end{array}$ & $1200-1300$ & UK & $\begin{array}{l}\text { Myanmar, China, } \\
\text { Thailand }\end{array}$ \\
\hline 07 & $\begin{array}{l}\text { Arthraxon hispidus } \\
\text { (Thunb.) Makino }\end{array}$ & Sept.-Oct. & 1700 & HP, UK & Bhutan, China \\
\hline 08 & $\begin{array}{l}\text { Arthraxon lanceolatus } \\
\text { (Roxb.) Hochst. }\end{array}$ & Aug.-Jan. & 2400 & HP, UK & Arabia, Nepal, China \\
\hline 09 & $\begin{array}{l}\text { Arthraxon lancifolius } \\
\text { (Trin.) Hochst. }\end{array}$ & Jul.-Oct. & $1500-2000$ & $\begin{array}{l}\mathrm{HP}, \mathrm{J} \& \mathrm{~K}, \\
\mathrm{UK}\end{array}$ & $\begin{array}{l}\text { Africa, Bhutan, } \\
\text { Myanmar, Sri Lanka, } \\
\text { China, Pakistan, } \\
\text { Malaysia }\end{array}$ \\
\hline 10 & $\begin{array}{l}\text { Arthraxon microphyllus } \\
\text { (Trin.) Hochst. }\end{array}$ & Sept. & $1200-1500$ & HP & Bhutan, Nepal \\
\hline 11 & $\begin{array}{l}\text { Arthraxon prionodes } \\
\text { (Steud.) Dandy }\end{array}$ & $\begin{array}{l}\text { May.- } \\
\text { Aug. }\end{array}$ & $600-3600$ & HP, J\&K & Africa, Bhutan \\
\hline 12 & Arthraxon santapaui Bor & $\begin{array}{l}\text { Aug.- } \\
\text { Sept. }\end{array}$ & 1600 & UK & - \\
\hline 13 & $\begin{array}{l}\text { Arthraxon submuticus } \\
\text { (Nees ex Steud.) Hochst. }\end{array}$ & Sept.-Oct. & $600-1800$ & $\mathrm{HP}$ & China \\
\hline 14 & $\begin{array}{l}\text { Bothriochloa bladhii } \\
\text { (Retz.) S.T. Blake }\end{array}$ & Apr.-Jun. & 950 & $\begin{array}{l}\mathrm{HP}, \mathrm{J} \& \mathrm{~K}, \\
\mathrm{UK}\end{array}$ & $\begin{array}{l}\text { Africa, Australia, } \\
\text { Bhutan, China, } \\
\text { Pakistan }\end{array}$ \\
\hline 15 & $\begin{array}{l}\text { Bothriochloa insculpta } \\
\text { (A.Rich.) A. Camus }\end{array}$ & $\begin{array}{l}\text { Sept.- } \\
\text { Jan. }\end{array}$ & 1500 & UK & Australasia, Europe \\
\hline 16 & $\begin{array}{l}\text { Bothriochloa ischaemum } \\
\text { (L.) Keng }\end{array}$ & Jul.-Oct. & 3600 & HP, UK & $\begin{array}{l}\text { Africa, Bhutan, } \\
\text { Europe, Nepal, } \\
\text { Pakistan }\end{array}$ \\
\hline 17 & $\begin{array}{l}\text { Bothriochloa kuntzeana } \\
\text { (Hack.) Henrard }\end{array}$ & $\begin{array}{l}\text { Sept.- } \\
\text { Dec. }\end{array}$ & 1500 & UK & Nepal \\
\hline 18 & $\begin{array}{l}\text { Bothriochloa pertusa (L.) } \\
\text { A. Camus }\end{array}$ & Jul.-Oct. & $1500-3500$ & $\begin{array}{l}\mathrm{HP}, \mathrm{J} \& \mathrm{~K}, \\
\mathrm{UK}\end{array}$ & $\begin{array}{l}\text { Afghanistan, Africa, } \\
\text { Sri Lanka, China, } \\
\text { Pakistan }\end{array}$ \\
\hline 19 & $\begin{array}{l}\text { Capillipedium assimile } \\
\text { (Steud.) A. Camus }\end{array}$ & Sept.-Jan. & 1400 & $\mathrm{HP}, \mathrm{UK}$ & $\begin{array}{l}\text { Australia, Bhutan, } \\
\text { Pakistan, South } \\
\text { Africa }\end{array}$ \\
\hline 20 & $\begin{array}{l}\text { Capillipedium huegelii } \\
\text { (Hack.) A.Camus }\end{array}$ & $\begin{array}{l}\text { Aug.- } \\
\text { Sept. }\end{array}$ & 1350 & UK & $\begin{array}{l}\text { Africa, Asia, } \\
\text { Myanmar }\end{array}$ \\
\hline 21 & $\begin{array}{l}\text { Capillipedium parviflorum } \\
\text { (R.Br.) Stapf }\end{array}$ & Jul.-Sept. & 1700 & $\begin{array}{l}\mathrm{HP}, \mathrm{J} \& \mathrm{~K}, \\
\mathrm{UK}\end{array}$ & Australia, Bhutan \\
\hline 22 & $\begin{array}{l}\text { Chrysopogon nodulibarbis } \\
\text { (Hochst. ex Steud.) } \\
\text { Henrard }\end{array}$ & $\begin{array}{l}\text { Dec.- } \\
\text { Feb. }\end{array}$ & $1000-1200$ & UK & Pakistan, Sri Lanka \\
\hline
\end{tabular}


94 Tribe Andropogoneae in Western Himalaya

\begin{tabular}{|c|c|c|c|c|c|}
\hline \multirow{2}{*}{$\begin{array}{l}\text { S. } \\
\text { No. }\end{array}$} & \multirow[t]{2}{*}{ Name of taxa } & \multirow{2}{*}{$\begin{array}{l}\text { Flowers } \\
\text { \& Fruits }\end{array}$} & \multirow{2}{*}{$\begin{array}{l}\text { Altitude } \\
\text { (m) }\end{array}$} & \multicolumn{2}{|c|}{ Distribution } \\
\hline & & & & $\begin{array}{l}\text { Western } \\
\text { Himalaya }\end{array}$ & World \\
\hline 23 & $\begin{array}{l}\text { Chrysopogon aciculatus } \\
\text { (Retz.) Trin. }\end{array}$ & $\begin{array}{l}\text { Aug.- } \\
\text { Sept. }\end{array}$ & 1000 & UK & $\begin{array}{l}\text { Bhutan, Myanmar, } \\
\text { Sri Lanka }\end{array}$ \\
\hline 24 & $\begin{array}{l}\text { Chrysopogon fulvus } \\
\text { (Spreng.) Chiov. }\end{array}$ & $\begin{array}{l}\text { Feb.- } \\
\text { May., Jul.- } \\
\text { Sept. }\end{array}$ & $1000-2000$ & $\begin{array}{ll}\mathrm{HP}, & \mathrm{J} \& \mathrm{~K}, \\
\mathrm{UK} & \end{array}$ & East Africa \\
\hline 25 & $\begin{array}{l}\text { Chrysopogon gryllus (L.) } \\
\text { Trin. }\end{array}$ & Jul.-Oct. & $1500-3500$ & $\begin{array}{l}\mathrm{HP}, \mathrm{J} \& \mathrm{~K}, \\
\mathrm{UK}\end{array}$ & Bhutan, Nepal \\
\hline 26 & $\begin{array}{l}\text { Chrysopogon serrulatus } \\
\text { Trin. }\end{array}$ & Jun.-Dec. & $0-1000$ & HP, UK & $\begin{array}{l}\text { Bhutan, Nepal, } \\
\text { Pakistan }\end{array}$ \\
\hline 27 & $\begin{array}{l}\text { Chrysopogon zizanioides } \\
\text { (L.) Roberty }\end{array}$ & Jul.-Nov. & 1000 & $\begin{array}{l}\mathrm{HP}, \\
\mathrm{J} \& \mathrm{~K}, \mathrm{UK}\end{array}$ & $\begin{array}{l}\text { Africa, Bhutan, } \\
\text { Myanmar, South } \\
\text { East Asia, Sri Lanka }\end{array}$ \\
\hline 28 & $\begin{array}{l}\text { Cymbopogon caesius } \\
\text { (Hook. \& Arn.) Stapf }\end{array}$ & $\begin{array}{l}\text { Aug.- } \\
\text { Sept. }\end{array}$ & 2600 & UK & Pakistan \\
\hline 29 & $\begin{array}{l}\text { Cymbopogon commutatus } \\
\text { (Steud.) Stapf }\end{array}$ & Apr.-Oct. & 1500 & $\mathrm{HP}$ & $\begin{array}{l}\text { Arabia, Africa, } \\
\text { South Asia }\end{array}$ \\
\hline 30 & $\begin{array}{l}\text { Cymbopogon distans } \\
\text { (Nees ex Steud.) W. } \\
\text { Watson }\end{array}$ & Jun.-Oct. & $1500-3000$ & HP, UK & China \\
\hline 31 & $\begin{array}{l}\text { Cymbopogon flexuosus } \\
\text { (Nees ex Steud.) } \\
\text { W.Watson }\end{array}$ & Sept.-Oct. & $100-2200$ & UK & Myanmar, Thailand \\
\hline 32 & $\begin{array}{l}\text { Cymbopogon gidarba } \\
\text { (Buch.- Ham. ex Steud.) } \\
\text { A. Camus }\end{array}$ & $\begin{array}{l}\text { Aug.- } \\
\text { Sept. }\end{array}$ & 1800 & HP, UK & Myanmar \\
\hline 33 & $\begin{array}{l}\text { Cymbopogon jwaranscusa } \\
\text { (Jones) Schult. }\end{array}$ & $\begin{array}{l}\text { Mar-- } \\
\text { May. }\end{array}$ & 3000 & $\begin{array}{l}\mathrm{HP}, \mathrm{J} \& \mathrm{~K}, \\
\mathrm{UK}\end{array}$ & $\begin{array}{l}\text { Africa, Bhutan, } \\
\text { Nepal, Sri Lanka }\end{array}$ \\
\hline 34 & $\begin{array}{l}\text { Cymbopogon jwarancusa } \\
\text { subsp. olivieri (Boiss.) } \\
\text { Soenarko }\end{array}$ & Jul.-Sept. & $0-3000$ & $\mathrm{HP}$ & Pakistan \\
\hline 35 & $\begin{array}{l}\text { Cymbopogon martini } \\
\text { (Roxb.) W.Watson }\end{array}$ & $\begin{array}{l}\text { Aug.- } \\
\text { Nov., } \\
\text { Feb.- } \\
\text { May. }\end{array}$ & $1400-1500$ & $\begin{array}{l}\mathrm{HP}, \mathrm{J} \& \mathrm{~K}, \\
\mathrm{UK}\end{array}$ & Bhutan \\
\hline 36 & $\begin{array}{l}\text { Cymbopogon microstachys } \\
\text { (Hook.f.) Soenarko }\end{array}$ & Jul.- Sept. & 1200 & UK & China \\
\hline 37 & $\begin{array}{l}\text { Cymbopogon nardus (L.) } \\
\text { Rendle }\end{array}$ & $\begin{array}{l}\text { Nov.- } \\
\text { Apr. }\end{array}$ & 1500 & HP, UK & Bhutan, China \\
\hline 38 & $\begin{array}{l}\text { Cymbopogon osmastonii } \\
\text { R. Parker }\end{array}$ & Nov.-Apr. & $480-3200$ & UK & Bangladesh \\
\hline 39 & $\begin{array}{l}\text { Cymbopogon pospischilii } \\
\text { (K. Schum.) C.E. Hubb. }\end{array}$ & $\begin{array}{l}\text { Sept.- } \\
\text { Nov., } \\
\text { Mar.-Apr. }\end{array}$ & 1400 & HP, UK & China, Pakistan \\
\hline
\end{tabular}




\begin{tabular}{|c|c|c|c|c|c|}
\hline \multirow{2}{*}{$\begin{array}{l}\text { S. } \\
\text { No. }\end{array}$} & \multirow[t]{2}{*}{ Name of taxa } & \multirow{2}{*}{$\begin{array}{l}\text { Flowers } \\
\text { \& Fruits }\end{array}$} & \multirow{2}{*}{$\begin{array}{l}\text { Altitude } \\
\text { (m) }\end{array}$} & \multicolumn{2}{|c|}{ Distribution } \\
\hline & & & & $\begin{array}{l}\text { Western } \\
\text { Himalaya }\end{array}$ & World \\
\hline 40 & $\begin{array}{l}\text { Cymbopogon } \\
\text { schoenanthus (L.) Spreng. }\end{array}$ & Mar.-Jun. & 1500 & HP & - \\
\hline 41 & $\begin{array}{l}\text { Dichanthium annulatum } \\
\text { (Forssk.) Stapf }\end{array}$ & Feb.-Dec. & $1000-1800$ & $\begin{array}{l}\mathrm{HP}, \mathrm{J} \& \mathrm{~K}, \\
\mathrm{UK}\end{array}$ & $\begin{array}{l}\text { Africa, Australia, } \\
\text { Bhutan, China, } \\
\text { Myanmar, Indonesia }\end{array}$ \\
\hline 42 & $\begin{array}{l}\text { Dichanthium foulkesii } \\
\text { (Hook.f.) S.K. Jain \& } \\
\text { Deshp. }\end{array}$ & Sept.-Oct. & 2000 & UK & Africa, China \\
\hline 43 & $\begin{array}{l}\text { Dichanthium foveolatum } \\
\text { (Delile) Roberty }\end{array}$ & $\begin{array}{l}\text { Aug.- } \\
\text { Nov. }\end{array}$ & 1800 & HP, J\&K & $\begin{array}{l}\text { Ethiopia, Iran, Sri } \\
\text { Lanka, Tanzania }\end{array}$ \\
\hline 44 & Dimeria ornithopoda Trin. & $\begin{array}{l}\text { Sept.- } \\
\text { Nov. }\end{array}$ & $460-600$ & UK & $\begin{array}{l}\text { Australia, Japan, } \\
\text { Malaya, Myanmar, } \\
\text { Nepal }\end{array}$ \\
\hline 45 & $\begin{array}{l}\text { Eulalia contorta (Brongn.) } \\
\text { Kuntze }\end{array}$ & Sept.-Oct. & 1800 & UK & $\begin{array}{l}\text { Australia, Bhutan, } \\
\text { China, Malaysia }\end{array}$ \\
\hline 46 & $\begin{array}{l}\text { Eulalia fimbriata (Hack.) } \\
\text { Kuntze }\end{array}$ & Oct.-Feb. & $300-1200$ & $\mathrm{HP}$ & Myanmar \\
\hline 47 & $\begin{array}{l}\text { Eulalia hirtifolia (Hack.) } \\
\text { A. Camus }\end{array}$ & Aug.-Oct. & $1500-3500$ & HP, UK & - \\
\hline 48 & $\begin{array}{l}\text { Eulalia leschenaultiana } \\
\text { (Decne.) Ohwi }\end{array}$ & Dec.-Feb. & $1600-2400$ & UK & $\begin{array}{l}\text { Bhutan, China, } \\
\text { Philippines, Thailand }\end{array}$ \\
\hline 49 & $\begin{array}{l}\text { Eulalia madkotiensis } \\
\text { Kandwal, B.K. Gupta \& } \\
\text { S.K. Srivast. }\end{array}$ & $\begin{array}{l}\text { Aug.- } \\
\text { Sept. }\end{array}$ & 1500 & UK & - \\
\hline 50 & $\begin{array}{l}\text { Eulalia mollis (Griseb.) } \\
\text { Kuntze }\end{array}$ & Aug.-Oct. & $1500-3500$ & HP, UK & $\begin{array}{l}\text { Bhutan, China, } \\
\text { Nepal, Pakistan }\end{array}$ \\
\hline 51 & $\begin{array}{l}\text { Eulalia quadrinervis } \\
\text { (Hack.) Kuntze }\end{array}$ & Sept.-Oct. & 2600 & HP,UK & $\begin{array}{l}\text { Bhutan, Myanmar, } \\
\text { Nepal, China, } \\
\text { Thailand }\end{array}$ \\
\hline 52 & Eulalia staintonii Bor & $\begin{array}{l}\text { Aug.- } \\
\text { Sept. }\end{array}$ & $1500-2600$ & UK & Nepal \\
\hline 53 & $\begin{array}{l}\text { Eulalia trispicata (Schult.) } \\
\text { Henrard }\end{array}$ & $\begin{array}{l}\text { Sept.- } \\
\text { Nov. }\end{array}$ & $300-1500$ & HP, UK & $\begin{array}{l}\text { Bangladesh, Bhutan, } \\
\text { Myanmar, China, } \\
\text { Malaysia, Nepal, Sri } \\
\text { Lanka, Vietnam }\end{array}$ \\
\hline 54 & $\begin{array}{l}\text { Eulaliopsis binata (Retz.) } \\
\text { C.E. Hubb. }\end{array}$ & $\begin{array}{l}\text { May.- } \\
\text { Nov. }\end{array}$ & 2200 & $\begin{array}{l}\mathrm{HP}, \mathrm{J} \& \mathrm{~K}, \\
\mathrm{UK}\end{array}$ & $\begin{array}{l}\text { Afghanistan, Bhutan, } \\
\text { China, Myanmar, } \\
\text { Philippines, Thailand }\end{array}$ \\
\hline 55 & $\begin{array}{l}\text { Hackelochloa granularis } \\
\text { (L.) Kuntze }\end{array}$ & Jul.-Nov. & 1500 & $\begin{array}{l}\text { HP, J\&K, } \\
\text { UK }\end{array}$ & $\begin{array}{l}\text { Africa, Bhutan, } \\
\text { Myanmar, Sri Lanka }\end{array}$ \\
\hline 56 & $\begin{array}{l}\text { Hemarthria altissima } \\
\text { (Poir.) Stapf \& C.E. Hubb. }\end{array}$ & Nov.-Feb. & 500 & UK & China \\
\hline 57 & $\begin{array}{l}\text { Hemarthria compressa } \\
\text { (L.f.) R.Br. }\end{array}$ & $\begin{array}{l}\text { Aug.-- } \\
\text { Sept. }\end{array}$ & 1500 & $\begin{array}{l}\mathrm{HP}, \mathrm{J} \& \mathrm{~K}, \\
\mathrm{UK}\end{array}$ & Bhutan \\
\hline
\end{tabular}




\begin{tabular}{|c|c|c|c|c|c|}
\hline \multirow{2}{*}{$\begin{array}{l}\text { S. } \\
\text { No. }\end{array}$} & \multirow[t]{2}{*}{ Name of taxa } & \multirow{2}{*}{$\begin{array}{l}\text { Flowers } \\
\text { \& Fruits }\end{array}$} & \multirow{2}{*}{$\begin{array}{l}\text { Altitude } \\
\text { (m) }\end{array}$} & \multicolumn{2}{|c|}{ Distribution } \\
\hline & & & & $\begin{array}{l}\text { Western } \\
\text { Himalaya }\end{array}$ & World \\
\hline 58 & $\begin{array}{l}\text { Heteropogon contortus } \\
\text { (L.)P. Beauv. ex Roem. \& } \\
\text { Schult. }\end{array}$ & Jun.-Dec. & $1800-3000$ & $\begin{array}{l}\mathrm{HP}, \quad J \& \mathrm{~K}, \\
\mathrm{UK}\end{array}$ & Bhutan \\
\hline 59 & $\begin{array}{l}\text { Heteropogon } \\
\text { melanocarpus (Elliott) } \\
\text { Benth. }\end{array}$ & $\begin{array}{l}\text { Aug.- } \\
\text { Sept. }\end{array}$ & $1000-1500$ & UK & China \\
\hline 60 & $\begin{array}{l}\text { Imperata cylindrica (L.) } \\
\text { Raeusch. }\end{array}$ & Jan.-Dec. & $1200-2500$ & $\begin{array}{l}\mathrm{HP}, \quad J \& \mathrm{~K}, \\
\mathrm{UK}\end{array}$ & Myanmar, Sri Lanka \\
\hline 61 & $\begin{array}{l}\text { Ischaemum polystachyum } \\
\text { J. Presl }\end{array}$ & Aug.-Jan. & $50-700$ & HP & Bangladesh \\
\hline 62 & $\begin{array}{l}\text { Ischaemum rugosum } \\
\text { Salisb. }\end{array}$ & Aug.-Oct. & 1400 & $\begin{array}{l}\mathrm{HP}, \quad \mathrm{J} \& \mathrm{~K}, \\
\mathrm{UK}\end{array}$ & $\begin{array}{l}\text { Africa, Bhutan, } \\
\text { China, Malaya, } \\
\text { Myanmar, Sri Lanka, } \\
\text { Thailand, China }\end{array}$ \\
\hline 63 & $\begin{array}{l}\text { Iseilema anthephoroides } \\
\text { Hack. }\end{array}$ & $\begin{array}{l}\text { Aug.- } \\
\text { Dec. }\end{array}$ & $400-425$ & UK & - \\
\hline 64 & Iseilema laxum Hack. & $\begin{array}{l}\text { Aug.- } \\
\text { Dec. }\end{array}$ & $1100-2750$ & UK & Sri Lanka \\
\hline 65 & $\begin{array}{l}\text { Microstegium ciliatum } \\
\text { (Trin.) A. Camus }\end{array}$ & $\begin{array}{l}\text { Aug.- } \\
\text { Nov. }\end{array}$ & $600-1500$ & $\begin{array}{l}\mathrm{HP}, \quad \mathrm{J} \& \mathrm{~K}, \\
\mathrm{UK}\end{array}$ & Bhutan, Myanmar \\
\hline 66 & $\begin{array}{l}\text { Microstegium delicatulum } \\
\text { (Hook.f.) A.Camus }\end{array}$ & Sept.-Oct. & $800-1600$ & UK & $\begin{array}{l}\text { Myanmar, China, } \\
\text { Thailand }\end{array}$ \\
\hline 67 & $\begin{array}{l}\text { Microstegium eucnemis } \\
\text { (Nees ex Steud.) A. Camus }\end{array}$ & Dec.-Jan. & $990-2300$ & UK & $\begin{array}{l}\text { Myanmar, China, } \\
\text { Thailand }\end{array}$ \\
\hline 68 & $\begin{array}{l}\text { Microstegium falconeri } \\
\text { (Hook.f.) Clayton }\end{array}$ & $\begin{array}{l}\text { Sept.- } \\
\text { Nov. }\end{array}$ & $1700-2500$ & HP, UK & Bhutan \\
\hline 69 & $\begin{array}{l}\text { Microstegium fasciculatum } \\
\text { (L.) Henrard }\end{array}$ & $\begin{array}{l}\text { Sept.- } \\
\text { Nov. }\end{array}$ & 1400 & UK & $\begin{array}{l}\text { Bhutan, Myanmar, } \\
\text { China, Nepal, } \\
\text { Thailand, Veitnam }\end{array}$ \\
\hline 70 & $\begin{array}{l}\text { Microstegium nudum } \\
\text { (Trin.) A. Camus }\end{array}$ & $\begin{array}{l}\text { Jun.- Jul., } \\
\text { Sept.- } \\
\text { Nov. }\end{array}$ & $1800-3600$ & $\mathrm{HP}, \mathrm{UK}$ & Bhutan, Nepal \\
\hline 71 & $\begin{array}{l}\text { Microstegium petiolare } \\
\text { (Trin.) Bor }\end{array}$ & $\begin{array}{l}\text { Sept.- } \\
\text { Dec. }\end{array}$ & $1500-2400$ & HP, UK & Myanmar, Nepal \\
\hline 72 & $\begin{array}{l}\text { Microstegium vimineum } \\
\text { (Trin.) A. Camus }\end{array}$ & Dec. & $1200-1500$ & $\mathrm{HP}, \mathrm{UK}$ & $\begin{array}{l}\text { Bhutan, China, } \\
\text { Japan, South East } \\
\text { Asia }\end{array}$ \\
\hline 73 & $\begin{array}{l}\text { Miscanthus fuscus (Roxb.) } \\
\text { Benth. }\end{array}$ & Apr.-Jun. & 2500 & UK & - \\
\hline 74 & $\begin{array}{l}\text { Miscanthus nepalensis } \\
\text { (Trin.) Hack. }\end{array}$ & $\begin{array}{l}\text { May-Jun., } \\
\text { Sept.-Oct. }\end{array}$ & $1500-3000$ & $\mathrm{HP}, \mathrm{UK}$ & Myanmar, Nepal \\
\hline 75 & $\begin{array}{l}\text { Mnesithea laevis (Retz.) } \\
\text { Kunth }\end{array}$ & $\begin{array}{l}\text { Sept-- } \\
\text { Oct. }\end{array}$ & 500 & $\begin{array}{l}\mathrm{HP}, \quad J \& \mathrm{~K}, \\
\mathrm{UK}\end{array}$ & $\begin{array}{l}\text { Bhutan, Indo- China, } \\
\text { Malay Islands, } \\
\text { Myanmar, Sri-Lanka }\end{array}$ \\
\hline
\end{tabular}




\begin{tabular}{|c|c|c|c|c|c|}
\hline \multirow{2}{*}{$\begin{array}{l}\text { S. } \\
\text { No. }\end{array}$} & \multirow[t]{2}{*}{ Name of taxa } & \multirow{2}{*}{$\begin{array}{l}\text { Flowers } \\
\text { \& Fruits }\end{array}$} & \multirow{2}{*}{$\begin{array}{l}\text { Altitude } \\
\text { (m) }\end{array}$} & \multicolumn{2}{|c|}{ Distribution } \\
\hline & & & & $\begin{array}{l}\text { Western } \\
\text { Himalaya }\end{array}$ & World \\
\hline 76 & $\begin{array}{l}\text { Ophiuros exaltatus }(\mathrm{L} .) \\
\text { Kuntze }\end{array}$ & Jul.-Feb. & $600-1000$ & UK & China \\
\hline 77 & $\begin{array}{l}\text { Phacelurus speciosus } \\
\text { (Steud.) C.E. Hubb. }\end{array}$ & Jul-Sept. & $1600-3500$ & $\begin{array}{l}\mathrm{HP}, \mathrm{J} \& \mathrm{~K}, \\
\mathrm{UK}\end{array}$ & - \\
\hline 78 & $\begin{array}{l}\text { Pogonatherum crinitum } \\
\text { (Thunb.) Kunth }\end{array}$ & Sept.-Oct. & 750 & HP, UK & $\begin{array}{l}\text { Afghanistan, } \\
\text { Myanmar, China, } \\
\text { Malaya }\end{array}$ \\
\hline 79 & $\begin{array}{l}\text { Pogonatherum paniceum } \\
\text { (Lam.) Hack. }\end{array}$ & Apr.-Nov. & $600-800$ & $\begin{array}{l}\mathrm{HP}, \mathrm{J} \& \mathrm{~K}, \\
\mathrm{UK}\end{array}$ & $\begin{array}{l}\text { Myanmar, Sri- } \\
\text { Lanka }\end{array}$ \\
\hline 80 & $\begin{array}{l}\text { Pogonatherum santapaui } \\
\text { Sur }\end{array}$ & $\begin{array}{l}\text { May-Oct., } \\
\text { Dec.-Mar. }\end{array}$ & $770-1400$ & UK & - \\
\hline 81 & $\begin{array}{l}\text { Polytrias indica (Houtt.) } \\
\text { Veldkamp }\end{array}$ & $\begin{array}{l}\text { Aug.- } \\
\text { Nov. }\end{array}$ & $2100-2400$ & UK & $\begin{array}{l}\text { Bangladesh, China, } \\
\text { Myanmar, Sri Lanka, } \\
\text { Vietnam }\end{array}$ \\
\hline 82 & $\begin{array}{l}\text { Pseudosorghum } \\
\text { fasciculare (Roxb.) A. } \\
\text { Camus }\end{array}$ & $\begin{array}{l}\text { Sept.- } \\
\text { Dec. }\end{array}$ & $1500-2300$ & UK & $\begin{array}{l}\text { Bhutan, Myanmar, } \\
\text { South East Asia }\end{array}$ \\
\hline 83 & $\begin{array}{l}\text { Rottboellia } \\
\text { cochinchinensis (Lour.) } \\
\text { Clayton }\end{array}$ & $\begin{array}{l}\text { Aug.- } \\
\text { Nov. }\end{array}$ & 1500 & UK & $\begin{array}{l}\text { Africa, Australia, } \\
\text { China, Malaya, Sri } \\
\text { Lanka }\end{array}$ \\
\hline 84 & $\begin{array}{l}\text { Rottboellia exaltata (L.) } \\
\text { L.f. }\end{array}$ & $\begin{array}{l}\text { Aug.- } \\
\text { Nov. }\end{array}$ & 600 & $\begin{array}{l}\text { HP, J\&K, } \\
\text { UK }\end{array}$ & China, Pakistan \\
\hline 85 & $\begin{array}{l}\text { Saccharum arundinaceum } \\
\text { Retz. }\end{array}$ & Aug.-Apr. & 1000 & UK & $\begin{array}{l}\text { Bangladesh, Bhutan, } \\
\text { Myanmar, Sri Lanka }\end{array}$ \\
\hline 86 & $\begin{array}{l}\text { Saccharum bengalense } \\
\text { Retz. }\end{array}$ & $\begin{array}{l}\text { Sept.- } \\
\text { Dec. }\end{array}$ & 1700 & $\begin{array}{l}\mathrm{HP}, \mathrm{J} \& \mathrm{~K}, \\
\mathrm{UK}\end{array}$ & - \\
\hline 87 & $\begin{array}{l}\text { Saccharum filifolium } \\
\text { Steud. }\end{array}$ & Apr.-Oct. & $1500-2500$ & HP, UK & - \\
\hline 88 & $\begin{array}{l}\text { Saccharum griffithii } \\
\text { Munro ex Aitch. }\end{array}$ & Jul.-Oct. & - & UK & $\begin{array}{l}\text { Afghanistan, } \\
\text { Pakistan }\end{array}$ \\
\hline 89 & $\begin{array}{l}\text { Saccharum longisetosum } \\
\text { (Andersson) V.Naray. ex } \\
\text { Bor }\end{array}$ & $\begin{array}{l}\text { Sept.- } \\
\text { May. }\end{array}$ & $1500-3000$ & HP, UK & $\begin{array}{l}\text { Myanmar, Nepal, } \\
\text { Bangladesh }\end{array}$ \\
\hline 90 & $\begin{array}{l}\text { Saccharum narenga (Nees } \\
\text { ex Steud.) Hack. }\end{array}$ & $\begin{array}{l}\text { Aug.- } \\
\text { Nov. }\end{array}$ & 800 & UK & Myanmar, Bhutan \\
\hline 91 & Saccharum officinarum L. & Jan.-Feb. & 1000 & UK & Africa \\
\hline 92 & $\begin{array}{l}\text { Saccharum ravennae }(\mathrm{L} .) \\
\text { L. }\end{array}$ & $\begin{array}{l}\text { Sept.- } \\
\text { Nov. }\end{array}$ & $1800-2700$ & $\begin{array}{l}\mathrm{HP}, \mathrm{J} \& \mathrm{~K}, \\
\mathrm{UK}\end{array}$ & Pakistan \\
\hline 93 & $\begin{array}{l}\text { Saccharum rufipilum } \\
\text { Steud. }\end{array}$ & Jun.-Dec. & $800-2100$ & $\begin{array}{l}\mathrm{HP}, \mathrm{J} \& \mathrm{~K}, \\
\mathrm{UK}\end{array}$ & Myanmar \\
\hline 94 & Saccharum spontaneum L. & Aug.-Jan. & $1000-1500$ & $\begin{array}{l}\mathrm{HP}, \mathrm{J} \& \mathrm{~K}, \\
\mathrm{UK}\end{array}$ & $\begin{array}{l}\text { Africa, Australia, } \\
\text { Myanmar, South } \\
\text { Europe, Sri Lanka }\end{array}$ \\
\hline
\end{tabular}


Tribe Andropogoneae in Western Himalaya

\begin{tabular}{|c|c|c|c|c|c|}
\hline \multirow{2}{*}{$\begin{array}{l}\text { S. } \\
\text { No. }\end{array}$} & \multirow[t]{2}{*}{ Name of taxa } & \multirow{2}{*}{$\begin{array}{l}\text { Flowers } \\
\text { \& Fruits }\end{array}$} & \multirow{2}{*}{$\begin{array}{l}\text { Altitude } \\
\text { (m) }\end{array}$} & \multicolumn{2}{|c|}{ Distribution } \\
\hline & & & & $\begin{array}{l}\text { Western } \\
\text { Himalaya }\end{array}$ & World \\
\hline 95 & $\begin{array}{l}\text { Schizachyrium brevifolium } \\
\text { (Sw.) Buse }\end{array}$ & $\begin{array}{l}\text { Sept.- } \\
\text { Dec. }\end{array}$ & 1500 & UK & $\begin{array}{l}\text { Africa, Bhutan, } \\
\text { Nepal, Sri Lanka }\end{array}$ \\
\hline 96 & $\begin{array}{l}\text { Schizachyrium impressum } \\
\text { (Hack.) A. Camus }\end{array}$ & $\begin{array}{l}\text { Aug.- } \\
\text { Sept. }\end{array}$ & 1200 & $\mathrm{~J} \& \mathrm{~K}, \mathrm{UK}$ & Pakistan \\
\hline 97 & $\begin{array}{l}\text { Schizachyrium exile } \\
\text { (Hochst.) Pilg. }\end{array}$ & $\begin{array}{l}\text { Sept.- } \\
\text { Dec. }\end{array}$ & $200-1500$ & UK & $\begin{array}{l}\text { Ethiopia, South } \\
\text { Africa }\end{array}$ \\
\hline 98 & $\begin{array}{l}\text { Sehima nervosum (Rottler) } \\
\text { Stapf }\end{array}$ & Jul.-Oct. & $>200$ & UK & $\begin{array}{l}\text { Australia, Myanmar, } \\
\text { China, Pakistan, Sri } \\
\text { lanka, Thailand }\end{array}$ \\
\hline 99 & $\begin{array}{l}\text { Sehima notatum (Hack.) } \\
\text { A. Camus }\end{array}$ & $\begin{array}{l}\text { Aug.- } \\
\text { Dec. }\end{array}$ & 2300 & $\mathrm{HP}, \mathrm{UK}$ & - \\
\hline 100 & $\begin{array}{l}\text { Sorghum arundinaceum } \\
\text { (Desv.) Stapf }\end{array}$ & $\begin{array}{l}\text { Sept.- } \\
\text { Dec. }\end{array}$ & $400-3000$ & $\mathrm{~J} \& \mathrm{~K}, \mathrm{UK}$ & $\begin{array}{l}\text { Australia, Bhutan, } \\
\text { Bolivia, Brazil, } \\
\text { Pakistan, South } \\
\text { Africa }\end{array}$ \\
\hline 101 & $\begin{array}{l}\text { Sorghum bicolor (L.) } \\
\text { Moench }\end{array}$ & $\begin{array}{l}\text { Sept.- } \\
\text { Nov. }\end{array}$ & $1200-1600$ & $\mathrm{HP}, \mathrm{UK}$ & $\begin{array}{l}\text { Australia, Bhutan, } \\
\text { China, South } \\
\text { America, Taiwan }\end{array}$ \\
\hline 102 & $\begin{array}{l}\text { Sorghum halepense (L.) } \\
\text { Pers. }\end{array}$ & Mar.-Dec. & $1700-1900$ & $\begin{array}{l}\mathrm{HP}, \quad \mathrm{J} \& \mathrm{~K}, \\
\mathrm{UK}\end{array}$ & $\begin{array}{l}\text { Bangladesh, Sri } \\
\text { Lanka }\end{array}$ \\
\hline 103 & $\begin{array}{l}\text { Sorghum miliaceum } \\
\text { (Roxb.) Snowden }\end{array}$ & $\begin{array}{l}\text { Aug.- } \\
\text { Nov. }\end{array}$ & 750 & HP, UK & Pakistan \\
\hline 104 & $\begin{array}{l}\text { Sorghum nitidum (Vahl) } \\
\text { Pers. }\end{array}$ & $\begin{array}{l}\text { Aug.- } \\
\text { Dec. }\end{array}$ & 500 & $\mathrm{HP}, \mathrm{UK}$ & $\begin{array}{l}\text { Australia, Bhutan, } \\
\text { Indo- China, } \\
\text { Thailand }\end{array}$ \\
\hline 105 & $\begin{array}{l}\text { Spodiopogon cotulifer } \\
\text { (Thunb.) Hack. }\end{array}$ & $\begin{array}{l}\text { Sept.- } \\
\text { Nov. }\end{array}$ & $1600-2000$ & $\begin{array}{l}\mathrm{HP}, \quad \mathrm{J} \& \mathrm{~K}, \\
\mathrm{UK}\end{array}$ & China, Japan \\
\hline 106 & Spodiopogon dubius Hack. & Jul.-Aug. & $2500-3000$ & $\mathrm{HP}, \mathrm{UK}$ & China, Nepal \\
\hline 107 & $\begin{array}{l}\text { Themeda anathera (Nees } \\
\text { ex Steud.) Hack. }\end{array}$ & Jul.-Dec. & $1500-3500$ & $\begin{array}{l}\mathrm{HP}, \quad \mathrm{J} \& \mathrm{~K}, \\
\mathrm{UK}\end{array}$ & $\begin{array}{l}\text { China, Pakistan, Sri } \\
\text { Lanka }\end{array}$ \\
\hline 108 & $\begin{array}{l}\text { Themeda arundinacea } \\
\text { (Roxb.) A. Camus }\end{array}$ & $\begin{array}{l}\text { Sept.- } \\
\text { Nov. }\end{array}$ & 750 & $\mathrm{HP}, \mathrm{UK}$ & $\begin{array}{l}\text { Bhutan, Myanmar, } \\
\text { South East Asia }\end{array}$ \\
\hline 109 & $\begin{array}{l}\text { Themeda caudata (nees ex } \\
\text { Hook. \& Arn.) A.Camus }\end{array}$ & $\begin{array}{l}\text { Sept.- } \\
\text { May }\end{array}$ & 2000 & UK & Bhutan, Myanmar \\
\hline 110 & $\begin{array}{l}\text { Themeda gigantea (Cav.) } \\
\text { Hack. ex Duthie }\end{array}$ & $\begin{array}{l}\text { Sept.- } \\
\text { Apr. }\end{array}$ & $700-2000$ & UK & $\begin{array}{l}\text { Bangladesh, China, } \\
\text { Nepal, Thailand }\end{array}$ \\
\hline 111 & $\begin{array}{l}\text { Themeda strigosa (Ham. } \\
\text { ex Hook.f.) A. Camus }\end{array}$ & Nov.-Jan. & 2300 & UK & Bangladesh \\
\hline 112 & $\begin{array}{l}\text { Themeda quadrivalvis (L.) } \\
\text { kuntze }\end{array}$ & $\begin{array}{l}\text { Aug.- } \\
\text { Nov. }\end{array}$ & 2300 & UK & Bhutan \\
\hline 113 & Themeda triandra Forssk. & May-Oct. & $1500-3500$ & $\begin{array}{l}\mathrm{HP}, \quad \mathrm{J} \& \mathrm{~K}, \\
\mathrm{UK}\end{array}$ & $\begin{array}{l}\text { Bhutan, Myanmar, } \\
\text { Sri Lanka }\end{array}$ \\
\hline 114 & $\begin{array}{l}\text { Themeda villosa (Lam.) A. } \\
\text { Camus }\end{array}$ & $\begin{array}{l}\text { Aug.- } \\
\text { Nov. }\end{array}$ & 1400 & $\mathrm{HP}, \mathrm{UK}$ & Bhutan \\
\hline
\end{tabular}




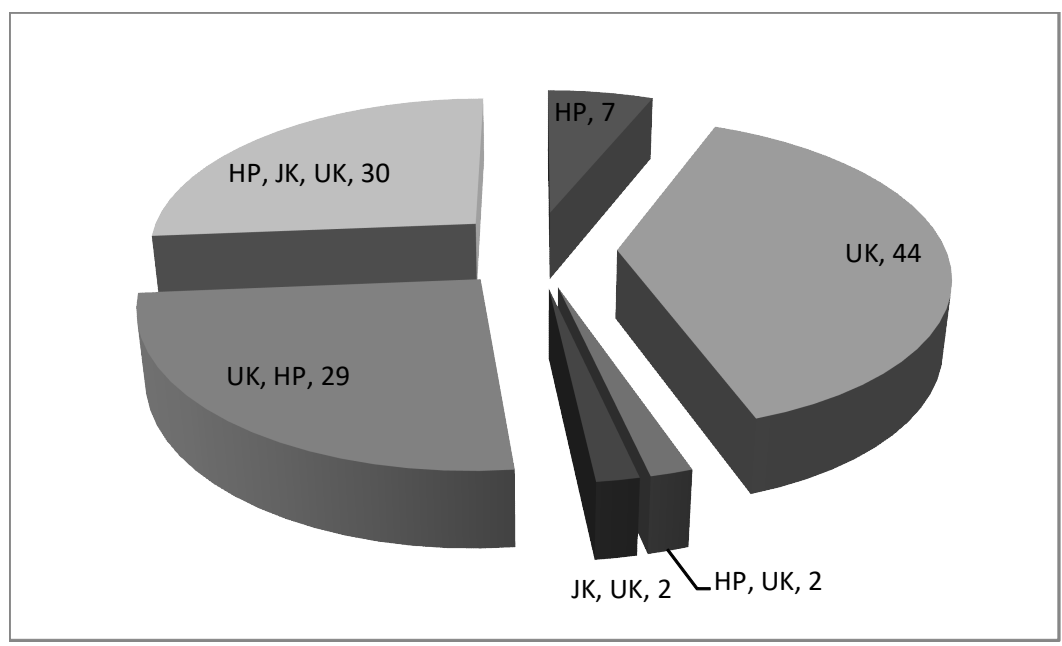

Figure 1. Distribution of taxa of Andropogoneae in different states of Western Himalaya

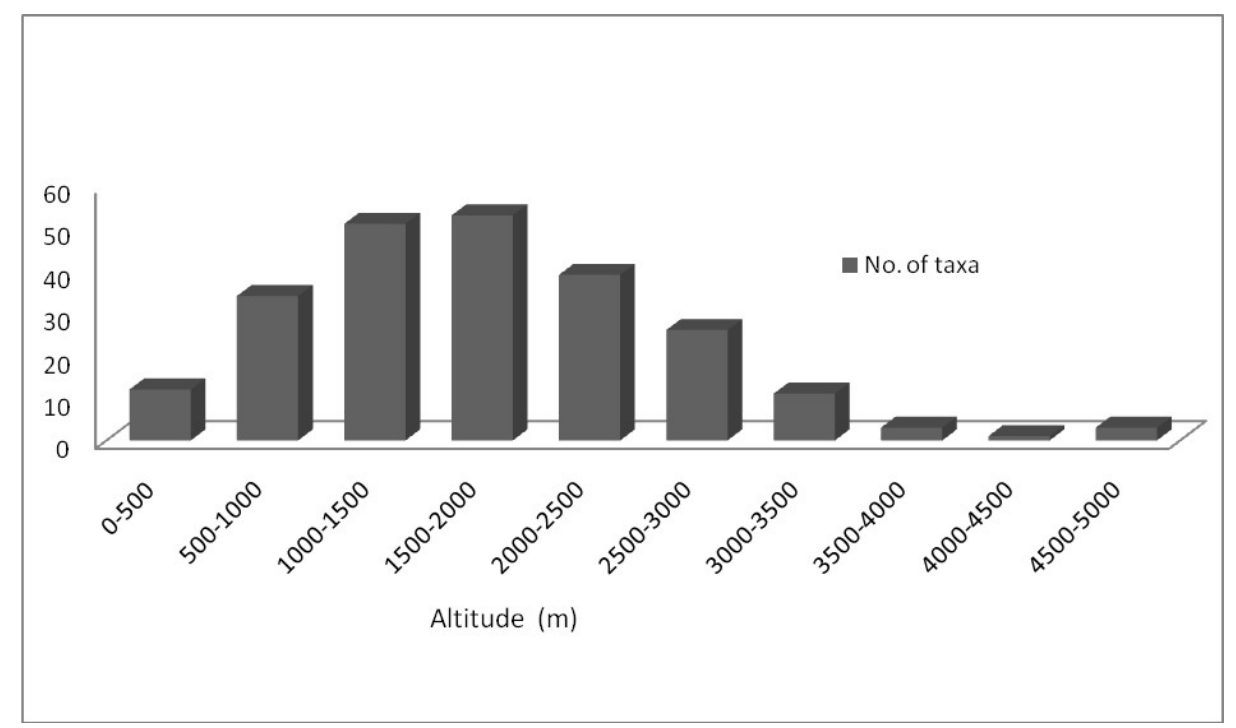

Figure 2. Altitudinal distribution of the tribe Andropogoneae in Western Himalaya

The distribution of the tribe in relation to altitude is depicted in Figure 2. Altitudinal gradient marks significant influence on diversity and distribution pattern of these grasses. Maximum diversity is found between an elevational range of $1500-2000 \mathrm{~m}$. Many taxa like Bothriochloa pertusa, Chrysopogon gryllus, Cymbopogon osmastonii, Eulalia mollis, Eulalia hirtifolia, Microstegium nudum, Themeda anathera, Themeda triandra, etc show a wide range of altitudinal distribution. On the other hand, few taxa like Arthraxon hispidus, Arthraxon lanceolatus, etc. are found in specific range which narrow down their altitudinal distribution. Very few taxa are found at an elevation of above $3500 \mathrm{~m}$. There have been reports suggesting that species richness is affected by increasing altitude as there has been reduction in diversity with increased elevation. This reduction could be attributed to eco-physiological constraints such as low temperature and other geographical barriers (Agnihotri et al. 2015). 
Table 2. Relative abundance of Andropogonoids

\begin{tabular}{|l|l|l|l|}
\hline Region & Genera & Species & References \\
\hline World & 85 & 1000 & Clayton et al., 1986 \\
\hline India & 63 & 350 & $\begin{array}{l}\text { Karthikeyan et al., } \\
1989\end{array}$ \\
\hline Western Himalaya & 33 & 113 & Present study \\
\hline
\end{tabular}

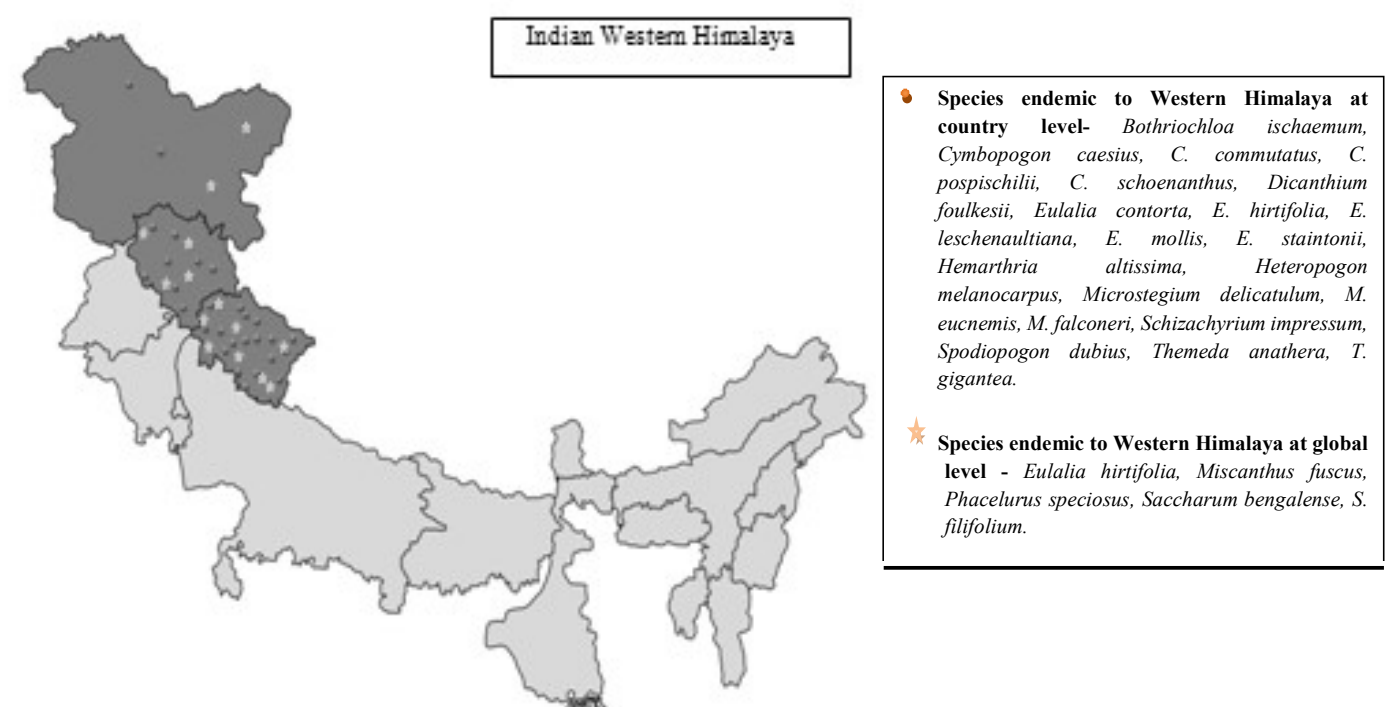

Figure 3. Distribution of endemic taxa of Andropogoneae in Western Himalaya

Out of the total taxa of Andropogonoid grasses, $46 \%$ is found to be strictly endemic to India especially to the Peninsular region (Mehrotra \& Jain 1980). Present study reveals that of all the taxa found in Western Himalaya, 7 taxa (6.14\%) are found to be endemic at global level (Figure 3) and 26 taxa $(22.80 \%)$ at Indian level. Interestingly, 19 taxa are such which are only found in western Himalaya and nowhere else in the country but are reported from other regions of the world as in China, Nepal, Pakistan, etc signifying their occurrence in the Himalayan region. Moreover, ten monotypic genera are found to occur in the region of Western Himalaya. Seven taxa of the tribe including Dimeria ornithopoda, Eulalia contorta, Hemarthria altissima, Hemarthria compressa, Imperata cylindrica, Pogonatherum paniceum and Schizachyrium brevifolium are included in IUCN red list of threatened plants which urges an immediate conservation implication.

\section{Acknowledgements}

Authors are thankful to the Director, CSIR-NBRI, Lucknow for his constant support and encouragement. 


\section{LITERATURE CITED}

Agnihotri, P.; Husain, D. \& Husain T. 2015. Assessment of diversity, endemism and distribution of the genus Aconitum L. ( Ranunculaceae) in India. Pleione. 9(1): 95 - 102.

Agnihotri, P. \& Husain, D. 2019. Revisionary studies on the genus Delphinium L. (Ranunculaceae) from India. In: P. Agnihotri \& J.S. Khuraijam (ed). Angiosperm Systematics: Recent Trends and Emerging Issues. BSMPS. Dehradun. ISBN : 978-81-211-0981-9. Pp. 1 - 56.

Bor, N.L. 1960. The Grasses of Burma, Ceylon, India and Pakistan (excluding Bambuseae). Pergamon Press. London. Pp. 767.

Brown, R. 1814. General remarks, geographical and systematical on the botany of the terra australis. In: M. Flinder (ed). A voyage to Terra Australis. Appendix 3. Pp. $580-$ 583.

Clayton, W.D. 1981. Notes on the tribe Andropogoneae (Gramineae). Kew Bull. 35: 813 818.

Clayton, W.D. 1987. Andropogoneae. In: T. R. Soderstrom, T.R.; Hilu, K.W.; Campbell C.S. \& Barkworth, M.E. (eds.), Grass systematics and evolution. Smithsonian Institution Press, Washington, D.C. Pp. 307 - 309.

Clayton, W.D. \& Renvoize, S.A. 1986. Genera Graminum. Kew Bull. Add. Ser. 13. Royal Botanic Gardens, Kew.

GPWG (Grass Phylogeny Working Group). 2001. Phylogeny and subfamilial classification of the Grasses (Poaceae). Ann. Missouri Bot. Gard. 88(3): 373 - 457.

Hackel, E. 1889. Andropogoneae. In: De Candolle, A. Monographiae Phanerogamarurn. 6. Paris.

Hartley, W. 1950. The global distribution of tribes of the Gramineae in relation to historical and environmental factors. Aust. Jour. Agr. Res. L. $1: 355-373$.

Hooker J.D. 1897. The Flora of British India. Vol. VII. L. Reeve \& Co. Ltd, London.

Husain, T.; Garg, A. \& Agnihotri, P. 2010. Genus Pedicularis L. (Scrophulariaceae) in India (A Revisionary Study). BSMPS. Dehradun. ISBN: 978-81-211-0735-8.

Jalal J.S. \& Jayanthi J. 2015. An annotated checklist of the orchids of Western Himalaya, India. Lankesteriana 15(1): 7 - 50.

Karthikeyan, S.; Jain, S.K.; Nayer, M.P.; \& Sanjappa, M. 1989. Florae Indicae Enumeration: Monocotyledonae. Botanical Survey of India. Calcutta

Kiran Raj, M.S. 2008. Taxonomic revision of the subtribe Dimeriinae Hack. of Andropogoneae in Peninsular India. Ph D. Thesis (unpublished). Calicut University.

Mehrotra, A. \& Jain S.K. 1980. Endemism in Indian grasses - Tribe Andropogoneae. BulL. Bot. Surv. Ind. 22: $51-58$.

Pachakkil, B.; Terajima, Y.; Ohmido, N.; Ebina, M.; Irei, S'; Hayashi, H. \& Takagi, H. 2019. Cytogenetic and agronomic characterization of intergeneric hybrids between Saccharum spp. hybrid and Erianthus arundinaceus . Scientific reports. 9(1):1748

Potdar, G.G.; Salunkhe, C.B. \& Yadav, S.R. 2012. Grasses of Maharashtra. Shivaji University, Kohlapur. Pp. 656.

Shukla, U. 1996. Grasses of North-Eastern India. Scientific publishers, Jodhpur. Pp. 404 
Simon, B.K. 2007. Grass Phylogeny and Classification: Conflict of Morphology and Molecules. Aliso: A J. Syst. Evol. Bot. 23 (1): 259 - 266.

Sreekumar, P.V. \& Nair, V.J. 1991. Flora of Kerala - Grasses in Flora of India (II). Botanical Survey of India, Kolkata. pp. 470.

The International Plant Names Index (2012). Published on the Internet http://www.ipni.org/ (accessed April, 2019).

The Plant List (2010). Version 1. Published on the Internet; http://www.theplantlist.org/ (accessed April, 2019)

Tropicos.org. (2016). Tropicos - Home. [online] Available at: http://tropicos.org/Home.aspx/ (accessed April, 2019)

Vegetti, A.C. 1998. Estudio fenetico de la inflorescencia en Andropogoneae (Poaceae). Kurtziana. 26: $145-160$.

Yadav S.R. 2010. Know your grass genera through hand lens. Shivaji University, Kohlapur. pp. 92. 